\title{
Guidelines of the Polish Society of Ophthalmology for the treatment of exudative age-related macular degeneration
}

\author{
Marta Misiuk-Hojło', Iwona Grabska-Liberek², Katarzyna Michalska-Małecka³, Ewa Mrukwa-Kominek ${ }^{3}$, Bożena \\ Romanowska-Dixon ${ }^{4}$, Marcin Stopa ${ }^{5}$, Jacek Szaflik ${ }^{6}$, Jerzy Szaflik ${ }^{6}$, Magdalena Ulińska ${ }^{6}$, Joanna E. Adamiec-Mroczek ${ }^{1}$ \\ 'Department and Clinic of Ophthalmology, Wroclaw Medical University, Wroclaw, Poland \\ ${ }^{2}$ Ophthalmology Department, Centre of Postgraduate Medical Education, Warsaw, Poland \\ ${ }^{3}$ Chair and Department of Ophthalmology, Medical University of Silesia, Katowice, Poland \\ ${ }^{4}$ Chair and Department of Ophthalmology, Jagiellonian University Medical College, Krakow, Poland \\ ${ }^{5}$ Department of Ophthalmology, Chair of Ophthalmology and Optometry, Heliodor Swiecicki University Hospital, Poznan University of Medical \\ Sciences, Poznan, Poland \\ ${ }^{6}$ Chair and Department of Ophthalmology, 2nd Faculty of Medicine, Medical University of Warsaw, Poland
}

\section{ABSTRACT}

Age-related macular degeneration (AMD) is the most common cause of severe visual impairment in older adults in the developed world. Especially neovascular AMD, characterized by rapid progression and a significant reduction in visual acuity, until recently was a therapeutic challenge. The last 15 years have been a period of development of new diagnostic and treatment possibilities. The paper presents recommendations of the Polish Ophthalmological Society for the management of neovascular age-related macular degeneration based on the latest publications.

KEY WORDS: wet AMD, anty-VEGF therapy, recommendations.

\section{AGE-RELATED MACULAR DEGENERATION}

Age-related macular degeneration (AMD) is the leading cause of significant and irreversible impairment of vision in the population over 50 years of age in highly developed countries. Age-related macular degeneration is a condition consisting of spontaneous changes - secondary to the ageing processes - involving the central retina (the macula). Factors increasing the risk of developing AMD include genetic disorders and living in northern regions of Europe. Smoking is considered as a modifiable risk factor. Consequently, patients with AMD or in the risk group for developing the disease are strongly advised against smoking.

\section{CHARACTERISTICS OF AGE-RELATED MACULAR DEGENERATION}

The early stages of AMD are characterized by the buildup of lipid deposits between the retinal pigment epithelium (RPE) and the Bruch's membrane. These accumulations of lipid material, referred to as drusen, appear ophthalmoscopi- cally as light-yellow foci of varying diameters. To support the diagnosis of AMD, the lesion size must be $\geq 63 \mu \mathrm{m}$. In addition to drusen, patients with AMD also develop morphological changes within the retinal pigment epithelium, which present as local hyper/hypopigmentation.

Drusen and RPE changes are classified as benign and typically do not cause impairment of central vision. The risk of disease progression into the dry form (geographic atrophy) and/or the exudative form depends on the type (i.e. hard/ soft), number and size of drusen identified by fundus examination, and ranges from $6.3 \%$ to $26 \%$ over a 5 -year period (mean determined in the AREDS study: 18\%). In patients with lesions in the RPE layer, an estimated $17.8 \%$ will develop advanced AMD. Aggravated lesions lead to a progressive and significant impairment in visual acuity.

Geographic atrophy (GA) is a sharply demarcated area of depigmentation associated with partial or complete atrophy of the retinal pigment epithelium followed by the outer layers of the sensory retina. Ophthalmoscopy shows large choroidal vessels present in the area corresponding to the atrophy of the outer retinal layers. Drusen and hypo/hyperpigmented lesions 
may surround a focus of atrophy. In this group of patients, a significant decrease in visual acuity occurs less commonly and progresses more slowly compared to patients with exudative AMD. Geographic atrophy may be complicated by the development of choroidal neovascularization, typically located on the border of the lesion.

The exudative form of AMD is also termed neovascular AMD. This disease entity is characterized by an uncontrolled growth of new blood vessels that originate from the choroid (choroidal neovascularization, CNV). Newly formed vascular plexuses penetrate through the Bruch's membrane into the space under the pigment epithelium (occult form, type $1 \mathrm{CNV}$ ) or the sensory retina (classic form, type $2 \mathrm{CNV}$ ). In some patients, the proliferation of neovascularization is present in both of these anatomical spaces. In such cases, a mixed form of CNV is diagnosed (type $4 \mathrm{CNV}$, dominantly or minimally classic type). Vascular proliferation observed in exudative AMD may also occur de novo in the macular region, initially from proliferating retinal vascular plexuses (RAP - retinal angiomatous proliferation, type $3 \mathrm{CNV}$ ). As the disease progresses, newly formed vessels merge with proliferating choroidal vessels to form chorioretinal anastomoses (CRA).

A common feature of all newly formed vessels are fenestrations through which blood and morphotic blood elements are able to penetrate into the surrounding tissues, causing detachment of the outer layers of the retina, edema, and sub- or intraretinal fluid accumulation. These morphological changes in the histological macular structure lead to damage to the outermost layers of the retina, frequently followed by local fibrotic processes. As a result, a central discoid scar is formed.

Another variant of AMD is idiopathic polypoidal choroidal vasculopathy (PCV), with primary abnormalities including dilated small choroidal vessels progressing to secondary aggressive forms of CNV.

Ophthalmoscopic signs of exudative age-related macular degeneration:

- neovascularization under the RPE or under the sensory retina visible as a grey green lesion;

- serous and/or hemorrhagic detachment of the neurosensory retina;

- serous and/or hemorrhagic detachment of the retinal pigment epithelium;

- hemorrhages - intraretinal, preretinal, rarely into the vitreous chamber;

- hard exudates within the macular region;

- progressive fibrotic processes observed subretinally or under the RPE.

Examinations used in the diagnostic work-up of exudative AMD are discussed in the section on therapy.
Differential diagnosis of exudative age-related macular degeneration:

- diabetic retinopathy;

- CNV associated with high myopia;

- CNV associated with inflammatory conditions, e.g. ocular histoplasmosis, punctate inner choroidopathy, retinal inflammatory scars;

- chronic serous chorioretinopathy (CSR), especially complicated by CNV;

- type 2 parafoveal telangiectasias.

\section{AIM OF TREATMENT OF EXUDATIVE AGE-RELATED MACULAR DEGENERATION}

Decrease in disease activity by reducing the amount of retinal fluid and modifying retinal anatomical parameters to improve or stabilize visual acuity in the long term.

\section{METHODS FOR TREATMENT OF EXUDATIVE AGE-RELATED MACULAR DEGENERATION}

A. Anti-VEGF therapy - intravitreal administration of drugs reducing the intraocular concentration of vascular endothelial growth factor (VEGF) - a pro-angiogenic protein increasing vascular permeability.

Anti-VEGF therapy is currently recognized as the gold standard in the treatment of exudative AMD and the first choice treatment for this condition.

\section{Currently used anti-VEGF drugs:}

1) Ranibizumab (Lucentis) - used in Poland since 2007.

Ranibizumab is a humanized monoclonal antibody fragment produced in Escherichia coli cells by applying recombinant DNA technology.

It has a capacity to bind to all VEGF-A isoforms.

The recommended single dose of the drug administered as an intravitreal injection is $0.05 \mathrm{ml}$, which corresponds to $0.5 \mathrm{mg}$ of the active substance ( $1 \mathrm{ml}$ of solution contains $10 \mathrm{mg}$ of ranibizumab).

Based on double-blind randomized clinical trials, the drug has been approved by the FDA and EMA for the treatment of:

- neovascular (exudative) form of age-related macular degeneration (AMD);

- visual impairment due to diabetic macular edema (DME);

- proliferative diabetic retinopathy (PDR);

- visual impairment due to macular edema secondary to retinal vein occlusion (RVO; branch RVO or central RVO);

- visual impairment due to choroidal neovascularization (CNV);

- retinopathy of prematurity (ROP) with zone I (stage $1+, 2+, 3$ or $3+$ ), zone II (stage $3+$ ) or AP-ROP (aggressive posterior ROP) disease. 
2) Aflibercept (Eylea) - used in Poland since 2013.

Aflibercept is a fusion protein consisting of portions of human VEGF (vascular endothelial growth factor) receptors 1 and 2 extracellular domains fused to the Fc portion of human IgG1 and produced in Chinese hamster ovary (CHO) $\mathrm{K} 1$ cells by applying recombinant DNA technology.

It has a capacity to bind to all VEGF-A and VEGF-B isoforms and the placental growth factor (PGF).

A single dose of aflibercept is $2 \mathrm{mg}$ contained in $0.05 \mathrm{ml}$ of solution ( $1 \mathrm{ml}$ of product contains $40 \mathrm{mg}$ of aflibercept).

Eylea is indicated for the treatment of patients with:

- neovascular (exudative) age-related macular degeneration (AMD);

- visual impairment due to macular edema secondary to retinal vein occlusion (BRVO, CRVO);

- visual impairment due to diabetic macular edema (DME);

- visual impairment due to myopic choroidal neovascularization (myopic CNV).

3) Brolucizumab (Beovu) - used in Poland since 2020.

Brolucizumab is a humanized monoclonal single-chain $\mathrm{Fv}(\mathrm{scFv})$ antibody fragment produced in Escherichia coli cells by applying recombinant DNA technology.

It has a capacity to bind to all VEGF-A types.

A single dose of brolucizumab is $6 \mathrm{mg}$ of the active substance contained in $0.05 \mathrm{ml}$ of injectable solution $(1 \mathrm{ml}$ contains $120 \mathrm{mg}$ of brolucizumab).

Beovu is a medicinal product indicated for the treatment of neovascular (exudative) form of age-related macular degeneration in adult patients.

4) Bevacizumab (Avastin) is a recombinant humanized monoclonal antibody produced in Chinese hamster ovary (CHO) cells by applying DNA technology.

The approved indications of bevacizumab include advanced oncological diseases. In ophthalmology, the drug is used on an off-label basis for the treatment of all diseases in which other anti-VEGF agents are used.

B. Photodynamic therapy (PDT) with verteporfin (Visudyne) is approved for the treatment of adult patients with exudative age-related macular degeneration (AMD), but only with predominantly classic CNV. Follow-up examinations (each time fluorescein angiography should be performed to precisely determine CNV borders) and treatments, if required, are performed at 3-month intervals based on the assessment of disease activity. In the treatment of exudative AMD, photodynamic therapy may be an alternative to anti-VEGF treatment in patients in whom - for various reasons - a therapy based on intravitreal injections may not be used. A modified procedure, called half-dosis PDT, can also be prescribed to patients with idiopathic polyploid choroidal vasculopathy, either as monotherapy or in com- bination with anti-VEGF treatment. Access to PDT is currently limited. This is because multiple medical centers have ceased to provide this therapy to their patients because of wider applications of anti-VEGF treatment.

C. Laser photocoagulation is not justified in most cases of AMD, as it shows a significantly lower efficacy compared to therapy with anti-VEGF agents. Laser photocoagulation can still be considered on a case-by-case basis for small CNV membranes away from the fovea.

D. Radiotherapy and surgery are not recommended in daily clinical practice because of their limited indications and efficacy.

The following part of the guidelines addresses only the commonly used anti-VEGF therapy.

Diagnosis of exudative AMD before and during antiVEGF therapy

1. General and ophthalmological history.

2. Best-corrected visual acuity (BCVA) determined using either the Snellen or ETDRS charts.

3. Dilated stereoscopic ophthalmoscopy.

4. Optical coherence tomography (OCT) - indicated directly before the start of treatment (maximum period: 60 days) and before each subsequent intravitreal injection.

5. OCT-angiography (angio-OCT) - a non-invasive imaging technique to visualize pathological choroidal and retinal blood vessels. In view of the static nature of the examination, evaluating the degree of disease activity is difficult and should also be based on the OCT analysis, visual acuity measurement, and patient history (the examination should be performed within 60 days before the start of treatment).

6. Fluorescein angiography (FA) - to determine the extent, type, size and location of CNV; FA is indicated in atypical cases (e.g. in patients with PCV) or diagnostically uncertain situations (before the start of treatment, not more than 60 days earlier).

7. Indocyanine angiography (ICG) - performed optionally in selected doubtful cases such as suspected PCV, massive serous PED or other abnormalities which yield inconclusive FA and OCT findings.

\section{Criteria of patient eligibility for anti-VEGF therapy}

1. Age over 45 years.

2. Best-corrected visual acuity of $0.05-0.9$, provided that impaired visual acuity is due to active CNV secondary to exudative AMD - under the NFZ drug programme "Treatment of age-related macular degeneration", patients with BCVA between 0.2 and 0.8 are treated. 
3. Presence of active choroidal neovascularization typical of the exudative form of AMD (including RAP, PCV).

4. Lesion location:

- subfoveal;

- parafoveal - if the lesion is active and carries the risk of foveal involvement and impaired visual acuity;

- extrafoveal - where there is a risk of vision loss;

5. Disease duration is a significant negative prognostic factor with regard to the final achievable visual acuity. At the same time, it is not a parameter that rules out anti-VEGF therapy in patients with AMD developing for many years and current $\mathrm{CNV}$ activity.

6. If there are indications for the treatment of both eyes, the interval between injections should be at least one week; the preferred option is to start treatment from the eye with better prognosis.

\section{Criteria of patient ineligibility for anti-VEGF therapy}

\section{A. Absolute}

1. Hypersensitivity to the active substance or to any of the excipients.

2. Active inflammatory changes within the globe of the eye and ocular adnexa.

3. History of recurrent uveitis.

4. Cataract preventing correct diagnosis.

5. Unregulated glaucoma or advanced glaucomatous neuropathy with the risk of significant progression due to temporary increases in intraocular pressure after intravitreal injections.

6. Dominant scarring involving the centre of the macula.

7. Dominant geographic atrophy involving the centre of the macula.

\section{B. Relative}

1. Ischemic stroke within the last 6 months.

2. Myocardial infarction within the last 6 months.

3. Other thromboembolic events within the last 6 months.

4. Treatment with anticoagulants if the INR exceeds 1.5 .

Patients taking anticoagulants should be informed about the increased risk of hemorrhagic complications: both spontaneous hemorrhages from $\mathrm{CNV}$ and abnormalities associated with the intravitreal procedure (e.g. subconjunctival or intravitreal hemorrhage). It should be noted, though, that general treatment takes priority over ophthalmic treatment, so interruptions in anticoagulant therapy are not recommended.

5. Ophthalmic procedure (cataract surgery or vitrectomy) performed during the preceding month.
In patients with advanced cataract and aggressive CNV, anti-VEGF treatment should not be discontinued in view of the risk of significant progression of lesions. It seems optimal to administer an antiVEGF drug 0-7 days before and 4 weeks after cataract surgery.

6. Dominant subretinal hemorrhage involving the center of the macula. In some cases, particularly when hemorrhage is identified in an extrafoveal location, continued anti-VEGF therapy may significantly improve the physical condition of the retina. In foveal hemorrhages, an alternative is tissue plasminogen activator (off-label use) into the vitreous space and anti-VEGF therapy and injection of gas (SF6) or vitrectomy (massive subretinal hemorrhage).

\section{Intravitreal injection procedures}

1. Intravitreal injection procedures should be performed by a qualified ophthalmologist with appropriate experience in administering intravitreal injections.

2. Appropriate local (drip) anesthesia must be provided.

3. The procedure should be performed under aseptic conditions, including topical administration of bactericidal substances with a broad spectrum of activity on the skin around the eye, the eyelid, and the surface of the eyeball. Antibiotic prophylaxis in the form of eye drops is not recommended either before or after the procedure because of the risk of developing antibiotic resistance to saprophytic strains in the human conjunctival sac.

4. Surgical hand disinfection, and the use of sterile gloves, sterile surgical drapes and sterile eyelid speculum.

5. The injection needle should be inserted into the central part of the vitreous chamber at a distance of $3.5 \mathrm{~mm}$ behind the corneal limbus in pseudo- or aphakic patients, and $4.0 \mathrm{~mm}$ in patients with an intact lens. The horizontal meridian should be avoided, and the injection should be aimed towards the centre of the globe. Subsequent injections, if possible, should be administered in other scleral sites.

6. After the intravitreal injection, the vascular perfusion status of the optic nerve disc should be evaluated (ophthalmoscopic examination), and intraocular pressure should be checked.

7. After the intravitreal injection procedure the patient should be instructed to report immediately any symptoms that might be suggestive of endophthalmitis (e.g. pain, redness of the eye, photophobia, blurred vision). 
Initial treatment phase (applies to all anti-VEGF drugs)

1. Three monthly initial injections performed at intervals of not less than four weeks;

2. Before each injection, the following should be performed:

- assessment of visual acuity,

- evaluation of intraocular pressure,

- slit lamp examination of the anterior segment of the eye,

- dilated indirect ophthalmoscopy,

- OCT.

3. Fluorescein angiography (FA) may be indicated if an unexplained significant decrease in BCVA is noted.

Continuation of treatment and patient monitoring after the initial three injections

At each follow-up visit, the following examinations should be done:

- assessment of visual acuity;

- measurement of intraocular pressure;

- slit lamp evaluation of the anterior segment of the eye;

- dilated indirect ophthalmoscopy;

- OCT;

- optionally fluorescein angiography in patients with an unexplained significant decrease in BCVA.

If no therapeutic effects are observed after five injections given at monthly intervals (i.e. the patient's visual acuity does not improve and/or the anatomical disease parameters remain unchanged) discontinuation of treatment or a change of drug should be considered.

\section{RECOMMENDED THERAPEUTIC REGIMENS}

Recommended therapeutic regimens are described in the SPCs of respective anti-VEGF agents. The proposed treatment procedures have been developed based on randomized clinical trials.

\section{Ranibizumab (Lucentis)}

After the initial treatment phase (with three consecutive monthly injections) the intervals between injections and follow-up evaluations should be determined individually for each patient by the physician in charge by determining CNV activity parameters (OCT, visual acuity, ophthalmoscopic examination). The most commonly used maintenance treatment regimen consists of intravitreal injection of ranibizumab following the identification of anatomical and functional changes indicative of disease recurrence - the so-called PRN (pro re nata) regimen.

A therapeutic protocol which prevents the recurrence of CNV activity is the so-called treat-and-extend (T\&E) regimen. Once maximum visual acuity has been regained and/or there are no signs of disease activity, the dosing in- terval can be gradually increased (typically by two weeks) until signs of disease activity and/or visual impairment are noted. The period between injections is then reduced by two weeks. In this therapeutic regimen, the patient receives an injection at each follow-up visit, also in the absence of CNV activity. It is currently considered that the interval between consecutive drug administrations should not exceed 12 weeks.

If the expected therapeutic benefit (improvement of vision, resolution of disease activity parameters determined by OCT) is not achieved, treatment should be changed to a different anti-VEGF drug.

\section{Aflibercept (Eylea)}

After three monthly injections to initiate therapy, the drug is administered on a fixed schedule every two months or T\&E until the end of the first year of treatment. According to the SPC, there is no requirement to monitor the patient's condition between consecutive injections during the first year of treatment. However, only periodic follow-ups provide an opportunity to determine whether the therapy is effective. During subsequent years of treatment, the interval between injections may be reduced or prolonged depending on CNV activity. Based on the physician's assessment of visual and/or anatomical parameters, a T\&E regimen may be initiated, whereby the dosing intervals are extended by two or four weeks to maintain the visual and/ or anatomical response in the patient. Intervals between injections longer than four months have not been studied.

If the patient's visual and/or anatomical parameters are found to have deteriorated, the dosing interval should be reduced accordingly to at least two months during the first year of treatment.

If the retinal functional and morphological outcomes (OCT) indicate that the patient is not benefiting from Eylea therapy, treatment should be discontinued or another drug should be prescribed.

\section{Brolucizumab (Beovu)}

After three loading doses, the physician may individualize treatment intervals depending on disease activity determined on the basis of visual acuity and/or anatomical parameters. An assessment of disease activity is suggested at 16 weeks (four months) after treatment start. In patients without disease activity, treatment every 12 weeks (three months) should be considered. In contrast, in patients with disease activity, treatment every eight weeks (two months) should be taken into consideration. In the clinical trials conducted in subjects selected for the 2-month regimen or in whom the 3-month regimen was reduced to two months during the period of follow-up, the option of introducing or returning to 3-month intervals was not considered. However, according to the SPC of the medicinal product, after three initial injections, the patient's physician may individualize the patient's treatment intervals based on dis- 
ease activity as assessed by follow-up examinations (visual acuity, OCT, ophthalmoscopy). The absence of therapeutic effects is an indication to discontinue brolucizumab therapy and shift to another anti-VEGF agent.

\section{CRITERIA FOR DISCONTINUATION OF THERAPY}

1. Lack of patient's consent to continue treatment.

2. Lack of benefit from treatment defined as no improvement in visual acuity and/or persistence of signs of disease activity despite 5-7 consecutive injections. In such a situation, a change to another anti-VEGF drug should be considered. An indication for the end of therapy there is no response to treatment with available drugs from the group of VEGF blockers.

3. Serious adverse drug reactions.

\section{DISCLOSURE}

The authors declare no conflict of interest.

\section{Piśmiennictwo}

1. Wong WL, Su X, Li Xi wsp. Global prevalence of age-related macular degeneration and disease burden projection for 2020 and 2040: a systematic review and meta-analysis. Lancet Glob Health 2014; 2: e106-116.

2. Curcio CA, Messinger JD, Sloan KR i wsp. Subretinal drusenoid deposits in non-neovascular age-related macular degeneration: morphology, prevalence, topography, and biogenesis model. Retina 2013; 33: 265-276.

3. Grzybowski A, Told R, Sacu Si wsp. 2018 Update on Intravitreal Injections: Euretina Expert Consensus Recommendations. Ophthalmologica 2018; 239: 181-193.

4. Ophthalmic Service Guidance Intravitreal injection therapy, The Royal College of Ophthalmologists.

5. Chew EY, Clemons T, SanGiovanni JP i wsp. The Age-Related Eye Disease Study 2 (AREDS2): study design and baseline characteristics (AREDS2 report number 1). Ophthalmology 2012; 119: 2282-2289.

6. Schmidt-Erfurth U, Waldstein SM. A paradigm shift in imaging biomarkers in neovascular age-related macular degeneration. Prog Retin Eye Res 2016; 50: 1-24.

7. Schmidt-Erfurth U, Chong V, Loewenstein A i wsp. Guidelines for the management of neovascular age-related macular degeneration by the European Society of Retina Specialists (EURETINA). Br J Ophthalmol 2014; 98: 1144-1167.

8. McClintic SM, Gao S, Wang J i wsp. Quantitative Evaluation of Choroidal Neovascularization under Pro Re Nata Anti-Vascular Endothelial Growth Factor Therapy with OCT Angiography. Ophthalmol Retina 2018; 2: 931-941.

9. Pilotto E, Frizziero L, Daniele AR i wsp. Early OCT angiography changes of type $1 \mathrm{CNV}$ in exudative AMD treated with anti-VEGF. Br J Ophthalmol 2019; 103: 67-71.

10. Schneider EW, Fowler SC. Optical coherence tomography angiography in the management of agerelated macular degeneration. Curr Opin Ophthalmol 2018; 29: 217-225.

11. Told R, Sacu S, Hecht A i wsp. Comparison of SD-optical coherence tomography angiography and indocyanine green angiography in type 1 and 2 neovascular age-related macular degeneration. Invest Ophthalmol Vis Sci 2018; 59: 2393-2400.

12. Maguire MG, Martin DF, Ying GS i wsp. Five-Year Outcomes with Anti-Vascular Endothelial Growth Factor Treatment of Neovascular Age-Related Macular Degeneration: The Comparison of Age-Related Macular Degeneration Treatments Trials. Ophthalmology 2016; 123: $1751-1761$.

13. Rofagha S, Bhisitkul RB, Boyer DS i wsp. Seven-year outcomes in ranibizumab treated patients in ANCHOR, MARINA, and HORIZON: a multicenter cohort study (SEVEN-UP). Ophthalmology 2013; 120: 2292-2299.

14. Guymer RH, Markey CM, McAllister IL i wsp. Tolerating Subretinal Fluid in Neovascular Age-Related Macular Degeneration Treated with Ranibizumab Using a Treat-and-Extend Regimen: FLUID Study 24-Month Results. Ophthalmology 2019; 126: 723-734.

15. Mantel I, Deli A, Iglesias K, Ambresin A. Prospective study evaluating the predictability of need for retreatment with intravitreal ranibizumab for age-related macular degeneration. Graefes Arch Clin Exp 0phthalmol 2013; 251: 697-704.

16. Richard G, Monés J, Wolf Si wsp. Scheduled versus pro re nata dosing in the VIEW trials. Ophthalmology 2015; 122: 2497-2503.

17. Singer MA, Awh CC, Sadda Si wsp. HORIZON:an open-label extension trial of ranibizumab for choroidal neovascularization secondary to age related macular degeneration. Ophthalmology 2012; 119: 1175-1183

18. Eleftheriadou M, Gemenetzi M, Lukic M i wsp. Three-Year Outcomes of Aflibercept Treatment for Neovascular Age-Related Macular Degeneration: Evidence from a Clinical Setting. Ophthalmol Ther 2018; 7:361-368.

19. Heier JS, Brown DM, Chong V i wsp. VIEW 1 and VIEW 2 Study Groups. Intravitreal aflibercept (VEGF trap-eye) in wet age-related macular degeneration. Ophthalmology 2012; 119: 2537-2548.

20. Martin DF, Maguire MG, Ying GS i wsp. Comparison of Age-related Macular Degeneration Treatments Trials (CATT) Research Group. Ranibizumab and bevacizumab for neovascular age-related macular degeneration. N Engl J Med 2011;364: 1897-1908.

21. Nguyen $\mathrm{CL}, \mathrm{Oh} \mathrm{LJ}$, Wong E i wsp. Anti-vascular endothelial growth factor for neovascular age-related macular degeneration: a meta-analysis of randomized controlled trials. BMC Ophthalmol 2018; 18: 130.

22. Dugel PU, Koh A, Ogura Y i wsp. HAWK and HARRIER: Phase 3, Multicenter, Randomized, Double-Masked Trials of Brolucizumab for Neovascular Age-Related Macular Degeneration. Ophthalmology 2020; 127: 72-84.

23. Dugel PU, Koh A, Ogura Y i wsp. HAWK and HARRIER: Phase 3, Multicenter, Randomized, Double-Masked Trials of Brolucizumab for Neovascular Age-Related Macular Degeneration. Ophthalmology 2020; 127: 72-84.

24. Charakterystyka produktu leczniczego Aflibercept.

25. Charakterystyka produktu leczniczego Beovu.

26. Charakterystyka produktu leczniczego Ranibizumab. 
27. Kiernan DF, Hariprasad SM, Rusu IM i wsp. Epidemiology of the association between anticoagulants and intraocular hemorrhage in patients with neovascular age-related macular degeneration. Retina 2010; 30: 1573-1578.

28. Kuhli-Hattenbach C, FischerlB, Schalnus $R$ i wsp. Subretinal hemorrhages associated with age-related macular degeneration in patients receiving anticoagulation or antiplatelettherapy. Am J Ophthalmol 2010; 149: 316-321.

29. Ysng SS, Fu AD, McDonald HR i wsp. Massive spontaneous choroidal hemorrhage. Retina 2003; 23: 139-144.

30. Shin JY, Lee JM, Byeon SH. Anti-vascular endothelial growth factor with or without pneumatic displacement for submacular hemorrhage. Am J Ophthalmol 2015; 159: 904-914.

31. Cheung CM, Bhargava M, Xiang L i wsp. Six-month visual prognosis in eyes with submacular hemorrhage secondary to age-related macular degeneration or polypoidal choroidal vasculopathy. Graefes Arch Clin Exp 0phthalmol 2013; 251: 19-25.

32. Hesse L, Schroeder B, Heller G, Kroll P. Quantitative effect of intravitreally injected tissue plasminogen activator and gas on subretinal hemorrhage. Retina 2000; 20:500-505.

33. American Academy of Ophthalmology; Age-Related Macular Degeneration Preferred Practice Pattern ${ }^{\circledR} 2020$. 